\title{
A Branch-and-Price Approach for a Ship Routing Problem with Multiple Products and Inventory Constraints
}

\author{
Rutger de Mare* \\ ORTEC, P.O. Box 490, NL-2800 AL Gouda, The Netherlands \\ rutger.demare@ortec.com \\ Remy Spliet \\ Econometric Institute, Erasmus University Rotterdam \\ P.O. Box 1738, NL-3000 DR Rotterdam, The Netherlands \\ spliet@ese.eur.nl \\ Dennis Huisman \\ Econometric Institute \& ECOPT, Erasmus University Rotterdam \\ P.O. Box 1738, NL-3000 DR Rotterdam, The Netherlands \\ huisman@ese.eur.nl
}

Econometric Institute Report EI2010-05

\begin{abstract}
In the oil industry, different oil components are blended in a refinery to fuel products. These products are transported to different harbors by ship. Due to the limited storage capacity at the harbors and the undesirability of a stock-out, inventory levels at the harbors have to be taken into account during the construction of the ship routes. In this paper, we give a detailed description of this problem, which we call the ship routing problem with multiple products and inventory constraints. Furthermore, we formulate this problem as a generalized set-covering problem, and we present a Branch-and-Price algorithm to solve it. The pricing problems have a very complex nature. We discuss a dynamic programming algorithm to solve them to optimality.
\end{abstract}

Keywords: ship routing, multiple-products, inventory constraints, set-partitioning formulation, column generation, branch-and-price

${ }^{*}$ Corresponding author 


\section{Introduction}

The main focus of an oil company is the production and sales of oil. At first sight, the distribution of the products is a secondary issue. Oil companies focused on exploration, production and refining. Since the refining equipment is very expensive, it is important to utilize this efficiently. However, the focus has changed since the seventies. Margins decreased when the market was deregulated and the OPEC got more powerful. Due to technological standardization, all players on the market were able to produce efficiently. Production was no longer the competitive element. Since the eighties, business processes and supply chain optimization have become more important. The economic crisis of 2008 gave further incentives to reduce costs.

At a refinery crude oil is separated into different components. Via (optional) conversion units, the components are blended into products: gas, naphtha, gasoline, kerosene, gas oil, etc. These components are blended into oil products called grades, which are stored in tanks. From the product tanks, grades are transported via primary terminals and secondary terminals to the customer. The transport from the refinery to the primary terminals located at a harbor is often done by ship. The large scale of the transported quantities implies that a lot of money is involved. The operating cost of a ship are typically between $\$ 50,000$ and $\$ 400,000$ a day. The inventory cost (including working capital cost) of one grade in one harbor is typically around $\$ 1$ million per year.

Inventory management can reduce the costs of supply substantially. The make to stock strategy changed to a make to order strategy. This strategy implies that a trade off should be made between inventory costs, economies of larger ship sizes and the risk of a stock-out. Ideally, ship routing decisions and inventory decisions should be a combined decision. These decisions can be very complex.

In this paper, we will consider such a ship routing problem with multiple products. Since the tanks at the different harbors have a limited capacity and stock-outs are highly undesirable, we have to take into account the inventory levels of the different grades at these harbors. This problem has been considered by several other authors, but mainly heuristic solution methods are presented. Ronen (2002) presents a two-stage solution approach for the multiple product ship-scheduling problems. First, it is determined which shipments have to be selected and afterwards, the different vessels are scheduled to transport these shipments. Al-Khayyal \& Hwang (2007) formulate the problem as a mixed integer linear program. They show that even small instances cannot be solved by a general purpose solver and they argue that specialized algorithms are needed. In this paper, we will develop a branchand-price algorithm.

Such an algorithm is presented earlier by Christiansen (1999) in the case of 
a single-product ship scheduling problem, and later by Brønmo et al. (2009) for a ship scheduling problem with flexible cargo sizes. Christiansen (1999) looks at the transportation of ammonia with production and consumption factories. She presents an exact branch-and-price approach to solve the problem. The difference with our problem is that she considers only one product, while oil consists of several grades. The different grades have to be transported in different compartments of a ship and can never be combined. As a consequence, the capacities of the different compartments have to be taken into account instead of the total capacity of a ship. Moreover, we need to take the different inventory levels of the different grades into account instead of only one total level.

The main contribution of our paper is the generalization of the exact solution approach presented by Christiansen (1999) to the case with multiple products such that it can be applied in the oil industry. We will leave it to future researchers to perform a computational study with this algorithm.

The remainder of this paper is organized as follows. In Section 2, we give a comprehensive description of the ship routing problem with multi-products and inventory constraints. We will formulate the problem as a generalized set partitioning problem, which is an extension of Christiansen's formulation for one product (see also Christiansen \& Nygreen (2005)), in Section 3. Afterwards in Section 4, we present a Branch-and-Price method, where the LP relaxation is solved by applying column generation in every node of the Branch-and-Bound tree. There are two different kind of pricing problems, one for every combination of harbor and grade, and one for every ship compartment. These pricing problems are much more complex to solve than in some well-known applications where column generation is applied (e.g. vehicle routing and crew scheduling, see Desrosiers et al. (1995); Desaulniers et al. (2005)). For both kind of pricing problems, we present a dynamic programming algorithm. Finally, we finish the paper with some concluding remarks and suggestions for further research in Section 5.

\section{Problem description}

The ship routing problem with multi-products and inventory constraints (SRPMPIC) can be shortly described as the problem of finding a feasible route for each ship, including an assignment of the different grades to the different compartments, taken into account the operational constraints of the ship and the inventory constraints of the different grades at the different harbors. We will now describe the SRPMPIC in more detail. 


\subsection{Ship properties}

Within maritime transportation, there are several modes of operation: liner, tramp and industrial operations, see Christiansen et al. (2004). Our problem can be categorized under industrial operators. This implies we will only consider owned ships, time and spot charters. We can describe the cost structure of these ships as a fixed price per time interval plus a fixed price for each trip.

Each ship has a number of compartments. These compartments have fixed sizes, i.e. the capacity of a compartment cannot be changed. The sizes of the different compartments in a ship may differ. Note that finding feasible allocations of grades to the compartments and ships becomes easier as the number of compartments per ship increases.

We define a trip as the direct connection from one harbor to another harbor with a certain vessel. A vessel is able to carry out a number of trips. Each ship has a fixed travel time for each trip. In the harbor, it takes some time to (un)load the vessel. This time depends on the quantity that is (un)loaded. We assume that the (un)loading time for a unit is fixed per grade per harbor. Furthermore, we assume that grades can be (un)loaded simultaneously.

A schedule for a harbor is defined by several arrivals of ships during the planning horizon. A specific arrival at a certain harbor is referred to as a harbor arrival. A ship visits several harbors during the planning horizon. A harbor can be visited by one ship more than once. If a ship arrives at a harbor too early, it is possible to wait until service can start at no extra costs. We will assume that each ship is available during the entire horizon.

\subsection{Harbor properties}

There are two types of harbors: (1) Load harbors that produce grades or receive grades from external sources. They can only load grades into the ships. (2) Discharge or unload harbors that consume grades or sell grades to external sources. They can only unload grades from the ships.

At each harbor (terminal) a number of grades is stored. The inventory level of the grades at the beginning of the planning period is given in advance. In planning phases, linear forecasts are often used to describe the demand. Therefore, we describe the decline with a fixed demand rate. The inventory level has some bounds that we will explain. The inventory level can never exceed the capacity of the tanks. The difference between the maximal inventory level and the actual inventory level is called the ullage. Tanks cannot be completely empty. The sediments on the bottom of the tanks are dirty residues. This part of the tank is called the dead stock or unpumpables and the associated level is the minimal inventory level. 
When a ship arrives to service the harbor, the inventory level increases with a certain speed: one unit per unloading time. It is assumed that only one ship at a time can stay in a harbor and a buffer time between two arrivals can be required, due to narrow channels, locks, or some kind of processing time in the harbor.

A ship can deliver several grades at a time. We can set a minimum and a maximum offload quantity for each grade. These bounds can be used when the operator has a trading deal with another company to deliver specified amounts of grades to certain (external) harbors.

The maximum number of arrivals is known in advance, because time slots for arrivals have to be bought before the scheduling process. From time slots, time windows for the start of service are determined. Also when the company has an agreement with another party to deliver specified amounts of grades, they agree to deliver them within a time window. If no time windows are specified, they can initially encompass the entire planning period for each arrival.

\section{Mathematical formulation}

We formulate the SRPMPIC as a set-partitioning problem. To simplify notation, we present a formulation where each ship is empty and ready for service at the beginning of the planning horizon at its initial position. In addition, all grades can be transported in all compartments. Note that the model can be easily extended to the situation where certain grades can only be transported in special compartments (e.g. refrigerated compartments). Also the costs of cleaning compartments after discharge are not included.

This set-partitioning formulation is, an extension of the formulation of Christiansen \& Nygreen (2005) for the single product ship scheduling problem, which was obtained by applying Dantzig-Wolfe decomposition to the arc-flow formulation of Christiansen (1999). Before we can present the mathematical formulation, we first have to introduce some notation.

Deliveries are made using ships $v \in V$, where $V$ is the set of ships. Each ship contains a positive number of compartments. The compartments are described by $c \in C_{v} \subseteq C$, where $C_{v}$ is the set of compartments in ship $v, C$ is the set of all compartments of all ships. $c_{v}^{*} \in C_{v}$ is defined as the first compartment in ship $v$. In addition, we define a compartment schedule $r \in R_{c}$, where $R_{c}$ is the set of compartment schedules for compartment $c$. A schedule $r$ includes information describing the harbors where the compartment is loaded or discharged, the quantities loaded and discharged and the arrival times.

Furthermore, we define harbors $i, j \in H_{T}$, where $H_{T}$ is the set of all harbors. The set of harbors that can be visited by ship $v$ is called $H_{v}$. Each 
harbor can be visited several times, at the arrivals $m, n \in M_{T i}$, where $M_{T i}$ is the set of all possible arrivals at harbor $i$. An arrival is described as a node $(i, m)$ for $i \in H_{T}$ and $m \in M_{T i}$. The set of arcs connecting arrivals that can be feasibly visited by ship $v$ in a single sequence is defined as $A_{v}$, with $\operatorname{arcs}(i, m, j, n) \in A_{v}$. The set of grades is denoted by $G$, indexed by $g$. The set $G_{H_{i}} \subseteq G$ is the set of grades that are produced or consumed in harbor $i$. Any combination of a harbor and a grade, will from now on be referred to as a harbor-grade. $S_{i g}$ is the set of sequences for each harbor-grade. A sequence $s \in S_{i g}$ is a feasible replenishment schedule to load or discharge a grade in a harbor (without any reference to particular compartments or ships). A sequence includes information on the quantities (un)loaded and the start times of the services.

We introduce two types of variables to indicate the use of a compartment schedule and a harbor-grade sequence. $\lambda_{c r} \in[0,1]$ represents the fraction that compartment $c$ uses schedule $r$. $\theta_{\text {igs }} \in[0,1]$ is the fraction sequence $s$ is used for harbor-grade $(i, g)$. Note that for instance $\lambda_{c r}=1$ means that compartment $c$ performs schedule $r$ completely. A combination of two compartment schedules is feasible if both schedules represent the same physical route, i.e. if they visit the same harbor arrivals in the same order. A convex combination of the arrival times and the quantities does not influence the feasibility of the schedule or sequence, because they may have non-integer values.

To convey the information associated with the schedule variables $\lambda_{c r}$ and sequence variables $\theta_{i g s}$ to the model, define the following parameters: $B_{\text {imcgr }}$ is equal to one if compartment $c$ serves grade $g$ in harbor $i$, at arrival $m$ in schedule $r$, and zero otherwise; $Y_{i m g s}$ is equal to one if grade $g$ is not loaded or unloaded in harbor $i$, at arrival $m$, in sequence $s . Q_{\text {imgcr }}^{C}$ is the quantity of grade $g$ (un)loaded by compartment $c$ in harbor $i$, at arrival $m$, in schedule r. $Q_{i m g s}^{H G}$ is the quantity of grade $g$ loaded or discharged in harbor-grade sequence $s$ in harbor $i$, at arrival $m$. $T_{i m g s}^{H G}$ denotes the time the service starts for sequence $s$ in harbor $i$, at arrival $m$ for grade $g$. $T_{i m c r}^{C}$ is the time the service starts for compartment $c$ in harbor $i$, at arrival $m$, if route $r$ is used. $T_{i m c r}^{C}$ is equal to zero if compartment $c$ does not visit harbor arrival $(i, m)$. $X_{\text {imjncr }}$ is equal to one if compartment $c$ sails directly from harbor $i$, arrival $m$ to harbor $j$, arrival $n$ in route $r$ and zero otherwise. To ensure that a convex combination of $X_{i m j n c r}$ induced by the values $\lambda_{c r}$ is binary, we introduce the auxiliary binary variables $x_{i m j n c r}$.

The compartment costs contain all transportation costs, including usage costs. We assign all costs of a route to the first compartment of the ship. We force all compartments to take the same route, so this does not influence the cost structure. Therefore, we denote $C_{c_{v}^{*} r}^{C}$ as the costs of sailing route $r$ for the first compartment of ship $v$ and $C_{i g s}^{H G}$ as the costs of selecting sequence $s$ for harbor-grade $(i, g)$. These costs can be written as: 


$$
\begin{aligned}
C_{c_{v}^{*} r}^{C} & =\sum_{i, m, j, n \in A_{v}} \alpha\left(B_{i m c_{v}^{*} r}, X_{i m j n c_{v}^{*} r}\right)+\sum_{i \in H_{T}} \sum_{m \in M_{T i}} \sum_{g \in G_{H i}} \beta_{i m c_{v}^{*} g} Q_{i m c_{v}^{*} g r}^{C}(1) \\
C_{i g s}^{H G} & =\sum_{m \in M_{T i}} \gamma\left(Y_{i m g s}\right)+\sum_{m \in M_{T i}} \delta_{i m g} Q_{i m g s}^{H G}
\end{aligned}
$$

where $\alpha$ and $\gamma$ represent arbitrary functions and $\beta_{i m c_{v}^{*} g}$ and $\delta_{i m g}$ are arbitrary constants. For other other cost structures, we cannot guarantee that the objective value of each feasible convex combination is calculated correctly.

Now, we can formulate the SRPMPIC as follows:

$$
\begin{aligned}
\min \sum_{v \in V} \sum_{r \in R_{c_{v}^{*}}} C_{c_{v}^{*} r}^{C} \lambda_{c_{v}^{*} r} & +\sum_{i \in H_{T}} \sum_{g \in G} \sum_{s \in S_{i g}} C_{i g s}^{H G} \theta_{i g s} \\
\sum_{r \in R_{c}} B_{i m c g r} \lambda_{c r}+\sum_{s \in S_{i g}} Y_{i m g s} \theta_{i g s} & \leq 1 \forall i \in H_{T}, m \in M_{T i}, g \in G_{H i}, c \in C \\
\sum_{c \in C} \sum_{r \in R_{c}} Q_{i m c g r}^{C} \lambda_{c r}-\sum_{s \in S_{i g}} Q_{i m g s}^{H G} \theta_{i g s} & =0 \forall i \in H_{T}, m \in M_{T i}, g \in G_{H i} \\
\sum_{v \in V} \sum_{r \in R_{c_{v}^{*}}} T_{i m c_{v}^{*} r}^{C} \lambda_{c_{v}^{*} r}-\sum_{s \in S_{i g}} T_{i m g s}^{H G} \theta_{i g s} & =0 \forall i \in H_{T}, m \in M_{T i}, g \in G_{H i} \\
\sum_{r \in R_{c_{v}^{*}}} T_{i m c_{v}^{*} r}^{C} \lambda_{c_{v}^{*} r}-\sum_{r \in R_{c}} T_{i m c r}^{C} \lambda_{c_{r}} & =0 \forall i \in H_{T}, m \in M_{T i}, v \in V, c \in C_{v}, c \neq\left(\mathbb{C}_{v}^{*}\right) \\
\sum_{r \in R_{c}} \lambda_{c r} & =1 \forall c \in C \\
\sum_{s \in S_{i g}} \theta_{i g s} & =1 \forall i \in H_{T}, g \in G_{H i} \\
\sum_{r \in R_{c}} X_{i m j n c r} \lambda_{c r} & =x_{i m j n c} \forall v \in V,(i, m, j, n) \in A_{v}, c \in C_{v}(10) \\
\lambda_{c r} & \geq 0 \forall c \in C, r \in R_{c} \\
\theta_{i g s} & \geq 0 \forall i \in H_{T}, g \in G_{H i}, s \in S_{i g} \\
x_{i m j n c} & \in\{0,1\} \forall(i, m, j, n) \in A_{v}, c \in C_{v}
\end{aligned}
$$

The objective function minimizes the total sum of the sequence and schedule costs. We link the physical route of the compartments to the arrivals in the harbor-grades with constraints (4). These physical route constraints state that if grade $g$ is not served in harbor $i$ arrival $m$, no compartment of any ship should serves grade $g$ at this harbor arrival. In a feasible solution, the quantity the compartments load or discharge at a service should be equal to the quantity the harbor-grade (un)loads. The quantity coupling constraints (5) force this behavior. In case there are no inventory restrictions 
for the load harbors, the equality sign can be replaced by a greater-equal sign. Time coupling constraints (6) force the times for the start of service in the compartment subproblem to be equal to the arrival times according to the harbor-grade sequences. Note that this is only forced for the first compartments of the ships. The compartments of one ship should sail the same physical route. In the ship compartment constraints (7), we force them to take the same route by restricting all compartments of the ship to be at the same place at the same time. Constraints (8), (9), (11) and (12) allow convex combinations of routes and sequences. When we take a convex combination of several routes, we force that the resulting routing decisions remain binary by using the integer route constraints (10). This is done using the auxiliary variable which is binary by constraints (13).

\section{An exact solution approach}

In section 3, the SRPMPIC has been formulated as a mixed integer programming problem. Unfortunately, the number of different feasible harbor-grade sequences and compartment routes (and thus variables) grows exponentially in the input size. Therefore, we present a branch-and-price approach where the linear programming relaxation at each node in the branching tree is solved with column generation. Branching decisions can be taken in the same way as normally by branching on the $x_{i m j n c}$ variables. The pricing problem is much more complicated here than in many other applications. Therefore, we devote the remainder of this section on the pricing problem, where we can distinguish two different subproblems: the harbor-grade subproblem and the compartment subproblem. To calculate the reduced costs, we define the following dual variables:

- $D_{i m c g}^{V}$ for constraints (4)

- $D_{i m g}^{Q}$ for constraints (5)

- $D_{i m g}^{T}$ for constraints (6)

- $D_{i m c}^{S}$ for constraints (7)

- $D_{c}^{\lambda}$ for constraints (8)

- $D_{i g}^{\theta}$ for constraints (9)

- $D_{i m j n c}^{X}$ for constraints (10)

The definition of the reduced costs for the harbor-grade subproblems differs from the definition for the compartment subproblem. Moreover, due to 
the restrictions where we make a difference between the first compartment of the ships and the other compartments, we have different definitions for these two types of compartments as well. The reduced costs for the harbor-grade subproblems are defined as:

$$
\overline{C_{i g s}^{H G}}=C_{i g s}^{H G}-\sum_{m \in M_{T i}} \sum_{c \in C} Y_{i m g s} D_{i m c g}^{V}+\sum_{m \in M_{T i}} Q_{i m g s}^{H G} D_{i m g}^{Q}+\sum_{m \in M_{T i}} T_{i m g s}^{H G} D_{i m g}^{T}-D_{i g}^{\theta}
$$

The reduced costs for the first compartments $c_{v}^{*}$ of the ships are defined as:

$$
\begin{aligned}
\overline{C_{c_{v}^{*} r}^{C}}= & C_{c_{v}^{*} r}^{C}-\sum_{i \in H_{T}} \sum_{m \in M_{T i}} \sum_{g \in G_{H i}} B_{i m c_{v}^{*} g r} D_{i m c_{v}^{*} g}^{V}-\sum_{i \in H_{T}} \sum_{m \in M_{T i}} \sum_{g \in G_{H i}} Q_{i m c_{v}^{*} g r}^{C} D_{i m g}^{Q} \\
& -\sum_{i \in H_{T}} \sum_{m \in M_{T i}} \sum_{g \in G_{H i}} T_{i m c_{v}^{*} r}^{C} D_{i m g}^{T}-\sum_{i \in H_{T}} \sum_{m \in M_{T i}} \sum_{c \in C_{v} \mid c \neq c_{v}^{*}} T_{i m c_{v}^{*} r}^{C} D_{i m c}^{S}-D_{c_{v}^{*}}^{\lambda} \\
& -\sum_{(i, m, j, n) \in A_{v}} X_{i m j n c_{v}^{*} r} D_{i m j n c_{v}^{*}}^{X}
\end{aligned}
$$

For the other compartments in the ships $c \in C_{v}, c \neq c_{v}^{*}$, the reduced costs are defined as:

$$
\begin{aligned}
\overline{C_{c r}^{C}}= & -\sum_{i \in H_{T}} \sum_{m \in M_{T i}} \sum_{g \in G_{H i}} B_{i m c g r} D_{i m c g}^{V}-\sum_{i \in H_{T}} \sum_{m \in M_{T i}} \sum_{g \in G_{H i}} Q_{i m c g r}^{C} D_{i m g}^{Q} \\
& +\sum_{i \in H_{T}} \sum_{m \in M_{T i}} T_{i m c r}^{C} D_{i m c}^{S}-D_{c}^{\lambda}-\sum_{(i, m, j, n) \in A_{v}} X_{i m j n c r} D_{i m j n c}^{X}
\end{aligned}
$$

In order to simply notation, from now we will use a linear cost function, which contains a fixed cost $\mu_{i m j n}$ for traveling on every $\operatorname{arc}(i, m, j, n) \in A_{v}$. Note that this cost is per definition equal to zero for any other compartment than the first compartment. Similarly, we introduce a linear cost function for the harbor-grade sequences. $\nu_{i m g}$ for the cost to serve grade $g$ in harbor $i$, at arrival $m$. In addition, we assume that the parameters $\beta_{i m c_{v}^{*}}$ and $\delta_{i m g}$ are equal to 0.

Now we are able to define a general expression for the reduced costs of all compartments:

$$
\begin{aligned}
\overline{C_{c r}^{C}}= & \sum_{(i, m, j, n) \in A_{v}}\left[\mu_{i m j n}-D_{i m j n c}^{X}\right] X_{i m j n c r} \\
& -\sum_{i \in H_{T}} \sum_{m \in M_{T i}}\left[\sum_{g \in G}\left(D_{i m c g}^{V} B_{i m c g r}\right)-\sum_{g \in G}\left[D_{i m g}^{Q} Q_{i m c g r}\right]-D_{i m c}^{S T} T_{i m c r}^{C}\right] \\
& -D_{c}^{\lambda}
\end{aligned}
$$


with

$$
D_{i m c}^{S T}= \begin{cases}\sum_{g \in G} D_{i m g}^{T}+\sum_{c^{\prime} \in C_{v}, c^{\prime} \neq c_{v}^{*}} D_{i m c^{\prime}}^{S}, & \text { if } c=c_{v}^{*} \\ D_{i m c}^{S}, & \text { otherwise. }\end{cases}
$$

In order to find the column with minimal reduced costs, we have to solve the harbor-grade subproblem, HGP for harbor-grade $(i, g)$ and the compartment sub-problem (CP) for each compartment $c$. These subproblems can be perceived as finding shortest paths in some dedicated networks.

\subsection{The harbor-grade subproblem}

\subsubsection{The harbor-grade network}

For each harbor-grade $(i, g)$, we define a network. In this network, the nodes are specified by the arrival number $m$ and the cumulative load quantity of each grade indexed by $p \in P$. So, the node is fully described by $(m, p)$. Observe that $p$ is the quantity (un)loaded before the start of the service at arrival $m$. Therefore, in every network, the first node is specified by $(1,0)$, nothing has been delivered yet at the first arrival. Note that additional nodes $(E, p)$ are also defined, their purpose will be discussed shortly.

An arc is defined for every two nodes $(m, p)$ and $(m+1, p+q)$, where $q$ represents the quantity (un)loaded at arrival $m$ and has a value between the minimum and maximum (un)load quantity. Additional arcs are defined from $(m, p)$ to $(E, p+q)$, usage of such an arc indicates that quantity $q$ is un(loaded) at the last arrival in the sequence, $m$. Observe that this network is acyclic.

As an example, suppose we have three possible (un)load quantities: 0, 20 and 40 . These are the quantities that can be loaded or discharged at harborgrade $(i, g)$. This implies that at the start of service $m=2$, we can have a cumulative (un)load quantity of 0,20 and 40. In this service we can (un)load again 0,20 or 40 , so the third service can start with a cumulative quantity of $0,20,40,60$ or 80 and so on. This example is illustrated in Figure 1, for four possible arrivals.

Some nodes and sequences can be excluded, because they imply a violation of the inventory constraints at the end of the planning horizon. These sequences can be determined by calculating the minimum quantity to be loaded or discharged, $Q_{i g}^{\mathrm{min}}$. This is the minimal quantity needed to prevent an inventory violation at the end of the horizon. When $Q_{p g}<Q_{i g}^{\min }$ this sequence can be deleted. With an analogue calculation, we can determine the maximum quantity to be loaded or discharged. So, we achieve the following inequalities: $Q_{i g}^{\min }<Q_{p g}<Q_{i g}^{\max }$. Only when $Q_{p g}$ is inside this interval, the node exists. In the example, node $(4,0)$ does not exist (indicated in the figure by that fact that no arcs are connected to this node). 
Figure 1: A harbor-grade network

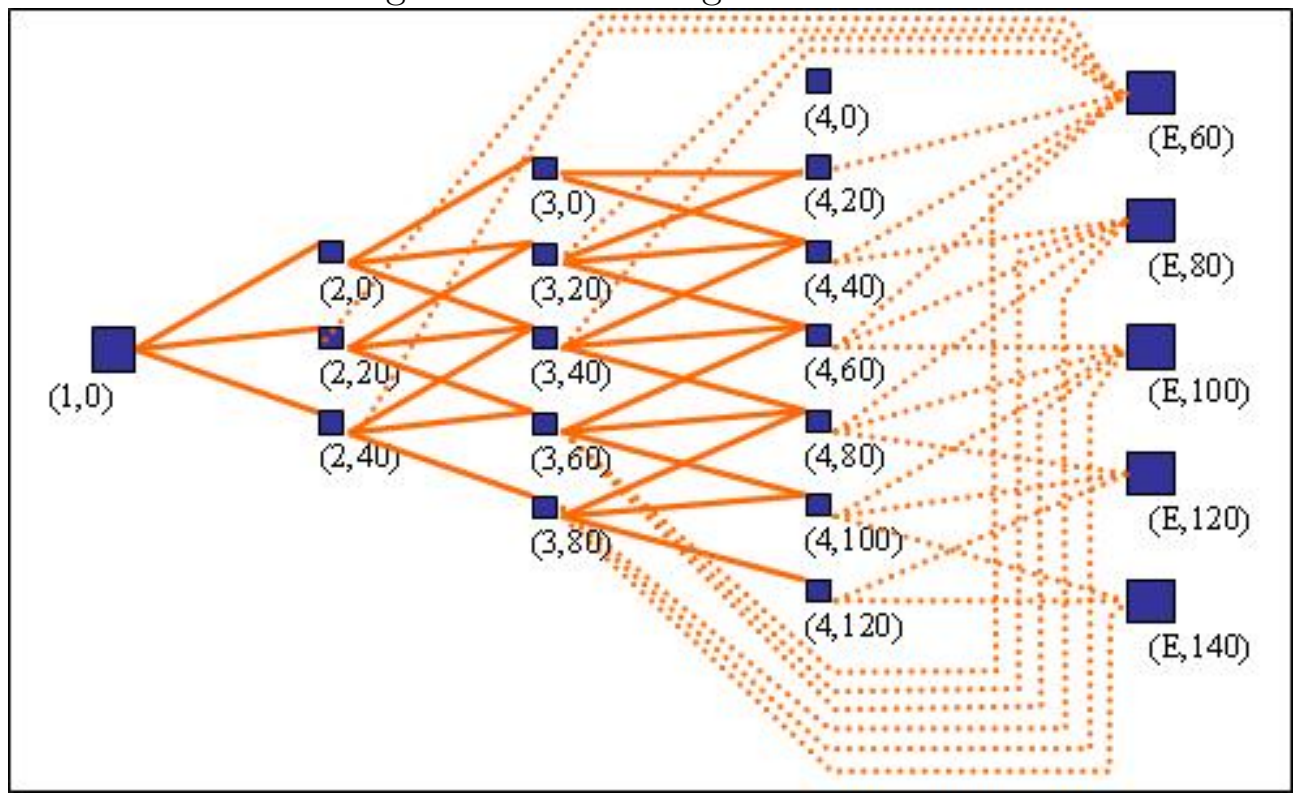

A path through the network from $(1,0)$ to $(E, p)$ for some p corresponds to a sequence of loads or discharges for a certain harbor-grade. When arrival times are determined for every node on that path, a harbor-grade sequence can be obtained. Obviously, we are only interested in feasible harbor-grade sequences, i.e. sequences which satisfy the time windows.

At each node the inventory level can be calculated depending on the time for the start of service, $t_{i m}$, with the demand rate, $R_{i g}$, the initial inventory level, $S_{i g}^{I}$, and the cumulative quantity loaded or discharged, $Q_{i g p s}$, before start of service in the node. Let $J_{i}$ be equal to 1 if harbor $\mathrm{i}$ is a load harbor and -1 if $i$ is a discharge harbor. The inventory constraints for each node in the network can now be formulated as follows:

$$
S_{i g}^{-} \leq S_{i g}^{I}-J_{i} Q_{i g p}-R_{i g} t_{i m} \leq S_{i g}^{+} \forall m \in M_{T i}, \forall p \in P
$$

From these constraints, we can derive time windows for start of service in the nodes as the inequalities only depend on the information of a single node. Therefore explicit inventory constraints are avoided.

Since convex combinations of sequences can be used as one sequence, any (un)load quantity between the minimum and maximum (un)load quantity can still be obtained if we discretize the (un)loaded quantities. Two aspects are important: the number of (un)loaded quantities we allow and the values we allow. Both decisions influence the calculation time when we solve the subproblem. To further limit the number of possible (un)loaded quantities for each arrival, and therefore limiting the number of arcs in the network, 'nice' values can be chosen for the (un)loaded quantities. We suggest to 
use equidistant quantities, because the number of nodes in the network can otherwise be exponential in $P$. Then for each next service, there are exactly $|P|-1$ new values for the cumulative quantity. So, arrival $m$ has $(|P|-$ $1)(m-1)+1$ nodes and the total network has a maximum number of nodes:

$$
\sum_{m=1}^{\left|M_{T i}\right|+1}[(|P|-1)(m-1)+1]=\frac{(|P|-1)\left|M_{T i}\right|+2}{2}\left(\left|M_{T i}\right|+1\right)
$$

\subsubsection{Constructing the harbor-grade subproblem}

Paths in the harbor-grade network and corresponding arrival times describe feasible sequences. To calculate these sequences, we need the following additional notation:

- $y_{i m g}$, which is 1 if node $(m, p)$ is not visited for any $p$ and 0 otherwise,

- $t_{i m g}$, which is the start time that node $(m, p)$ is served for a certain $p$. Note that a path can contain only one node $(m, p)$ for every $m$.

As $Q_{i g m p}^{H G}$ is the cumulative quantity loaded or discharged in harbor-grade $(i, g)$ before arrival at $(m, p)$, the quantity $q_{i m g}^{H G}$, when arc $((m-1, q),(m, p))$ is used, is now given by $\left|Q_{i g m p}^{H G}-Q_{i g m-1 q}^{H G}\right|$.

Using (14) and the explicit form of $C_{i g s}^{H G}$, a cost function is assigned to each sequence:

$$
\overline{C_{i g s}^{H G}}=\sum_{m \in M_{T i}}\left[\nu_{i m g} y_{i m g}-D_{i m c g}^{V} y_{i m g}+D_{i m g}^{Q} q_{i m g}^{H G}+D_{i m g}^{T} t_{i m g}\right]-D_{i g}^{\theta}
$$

Let $\left[A_{i g m p n q}^{H G}, B_{i g m p}^{H G}\right]$ be the time window for service at arrival $m$. As has been noted before, the time window depends on the inventory level. For a discharge harbor the time window for the start of service can only open after the moment in time that the inventory level at the end of service would not exceed the maximum inventory level, $S_{i g}^{+}$. Furthermore, the time window closes when the inventory level would be the minimum inventory level, $S_{i g}^{-}$, when no service is provided. In addition, we define $T_{i g}^{L}$ as the time required to (un)load one unit of grade $g$ at harbor $i$. An analogue relationship holds for a load harbor. In general, for harbor $i$ the following hold:

$$
\begin{gathered}
A_{i g m p n q}^{H G}=\max \left\{T_{i m}^{S}, \frac{S_{i g}^{+}-S_{i g}^{I}+J_{i} Q_{i g n q}^{H G}}{R_{i g}}-J_{i}\left(Q_{i g n q}^{H G}-Q_{i g m p}^{H G}\right) T_{i g}^{L}\right\} \\
B_{i g m p}^{H G}=\min \left\{T_{i m}^{E}, \frac{S_{i g}^{-}-S_{i g}^{I}+J_{i} Q_{i g m p}^{H G}}{R_{i g}}\right\}
\end{gathered}
$$


Now, let $T_{i g m p n q}^{H G}$ be the minimum time between node $(m, p)$ and $(n, q)$ for harbor-grade $(i, g)$, based on the buffer time and (un)loading time. Given that the service in $(n, q)$ starts before $t$, it holds for the start of service $t^{\prime}$ at node $(m, p)$, that:

$$
A_{i g m p n q}^{H G} \leq t^{\prime} \leq \min \left\{B_{i g m p}^{H G}, t-T_{i g m p n q}^{H G}\right\}
$$

Next define the recursive function $f_{i g m q}^{H G}(t)$ as the minimum costs of harborgrade $(i, g)$ up to arrival at node $(m, q)$ if the service of this node can start within time $t$ :

$$
\begin{aligned}
f_{i g n q}^{H G}(t)=\min _{p}\left\{\min _{A_{i g n p m q}^{H G} \leq t^{\prime} \leq \min \left\{B_{i g n p}^{H G}, t-T_{i g n p m q}^{H G}\right\}}\{\right. & D_{i m g}^{Q}\left[Q_{i g n q}^{H G}-Q_{i g m p}^{H G}\right] \\
& \left.\left.+D_{i n g}^{T} t+F_{i g n}^{H G}+f_{i g m p}^{H G}\left(t^{\prime}\right)\right\}\right\},
\end{aligned}
$$

where $f_{i g 1 q}^{H G}(t)=0$ and $F_{i g n}^{H G}=\sum_{m^{\prime}=m+1}^{\left|M_{T i}\right|}\left(\nu_{i m^{\prime} g}-\sum_{c \in C} D_{i m^{\prime} g c}^{V}\right)$ for $\mathrm{n}=\mathrm{E}$ and $F_{i g n}^{H G}=0$ otherwise. Note that $f_{i g m q}^{H G}(t)$ is non-increasing in $t$ and that it is piecewise linear. The harbor-grade subproblem is now defined as:

$$
\min _{q} f_{i g E q}^{H G}(T)
$$

with $T$ the planning horizon.

\subsubsection{Solving the harbor-grade subproblem}

The piecewise linear recursive cost function can be generated by forward propagation. The costs at a moment in time $t$ is defined as the minimum over a set of linear functions induced by the possible paths up to a node. The cost functions $f_{i g m q}^{H G}(t)$ are constructed by adding line pieces iteratively when one of the linear functions becomes apparent. Let a line piece $l$ for node $(m, p)$ be defined by a linear function $l(t)$ and an interval on which the function is valid for arrival $m$ and quantity $p$. Now, we can present the pseudo code of the algorithm to calculate these costs functions:

Initialize: Set $f_{i g(1)(0)}^{H G}(t)=0$ for $t \in[0, T]$ and mark the corresponding line piece as 'unfinished'. Set $f_{\text {igmp }}^{H G}(t)=\infty$ for all other nodes $(m, p)$.

While there are 'unfinished' line pieces: select an 'unfinished' line piece. Let the node corresponding to the line piece be $(m, p)$. For all nodes $(n, q)$ that is connected to $(m, p)$ do:

- Determine the new linear function: $l^{\prime}(t)=F_{i g m}^{H G}+D_{i m g}^{Q}[q-p]+$ $D_{i m g}^{T} t^{\prime}+l(t)$. The interval on which this function is valid for arrival 
$n$ and quantity $q$ is determined by the interval of the line piece $l$ corrected for the minimal travel time between node $(m, p)$ and node $(n, q)$.

- If there exists a $t$ such that $l^{\prime}(t)$ is strictly less than $f_{i q n p}^{H G}(t)$ mark $l^{\prime}(t)$ as 'unfinished'. Redefine $f_{i g n p}^{H G}(t)=\min \left\{f_{i g n p}^{H G}(t), l^{\prime}(t)\right\}$ on the interval of $l^{\prime}$.

- Let $T^{\prime}$ be the end of the interval of line piece $l^{\prime}$. If there exists a $t \in\left[T^{\prime}, T\right]$ such that $l^{\prime}\left(T^{\prime}\right)$ is strictly less than $f_{i g n p}^{H G}(t)$, in particular on the interval $\left[T^{\prime}, T^{*}\right]$ define the line piece $l^{*}(t)=l^{\prime}(T)$ on the interval $\left[T^{\prime}, T^{*}\right]$. Mark $l^{*}(t)$ as 'unfinished' and redefine $f_{\text {ignp }}^{H G}(t)=$ $l^{*} T^{\prime}$ on the interval $\left[T^{\prime}, T^{*}\right]$.

- Mark the line piece $l$ as 'finished'.

When these costs functions are calculated, problem (26) can be solved by backtracking.

\subsection{The compartment subproblems}

\subsubsection{The compartment network}

The compartment subproblem is represented as a network for each compartment $c$. This network is similar to the Harbor-Grade network, but it has some additional features.

A node is specified by a harbor arrival $(i, m)$ and load level $p_{g}$ of grade $g$ on board the compartment when arriving at $(i, m)$. A node is fully described by $\left(i, m,\left\{p_{g} \mid g \in G\right\}\right)$, or shortly $\left(i, m, p_{g}\right)$. We start with the empty compartment in the initial position, which is a harbor arrival itself.

The network is a directed graph. An arc between two nodes represents a connection between those nodes if it is feasible with respect to time windows and travel times (see Christiansen \& Nygreen (1998)).

Figure 2 shows an example of a compartment network. This is a situation with two grades, three load levels $(0,20,40)$, an initial position $(1,2)$ and three other harbor arrivals: $(3,3),(2,1)$ and $(1,3)$. Harbor 1 is a load harbor, harbor 2 and 3 are discharge harbors. In a load harbor, we only allow the compartment to arrive empty.

Not every route that is valid according to the graph is a valid route in the original problem. Harbor arrival $(i, m)$ could be visited twice because there are several nodes to represent $(i, m)$. If the time windows are wide, it might be possible to visit a harbor arrival $(i, m)$, sail to another arrival $(j, n)$ and sail back to an other $(i, m)$ node. Note that routes with cycles will never be selected in the master problem. However, from an implementation point of view, it could be worthwhile to prevent generating routes with cycles. 
Figure 2: A compartment schedule

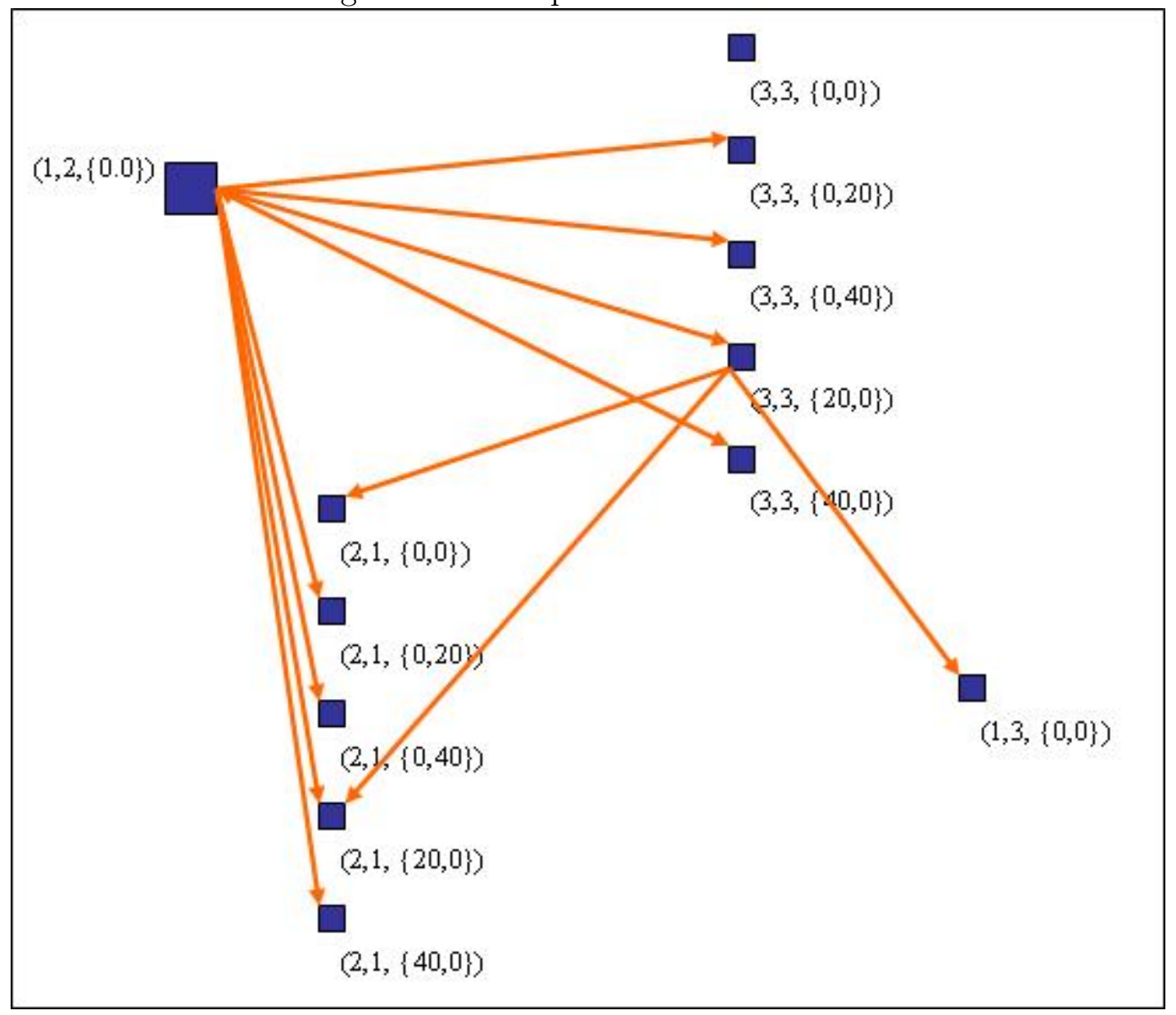


The ship can end its schedule in any node. This means we do not have to specify end-nodes as was done in the harbor-grade networks.

\subsubsection{Constructing the compartment subproblem}

The compartment network describes the feasible schedules. With the following additional notation:

- $Q_{i m p_{g} c}^{C}$ is the load level of grade $g$ in compartment $c$ when we arrive at $\left(i, m, p_{g}\right)$,

- $q_{i m c g}=\left|Q_{j n q_{g} c}^{C}-Q_{i m p_{g} c}^{C}\right|$, when we directly sail to $\left(j, n, q_{g}\right)$,

- $w_{i m c g}$ is 1 if compartment $c$ is allocated to grade $g$ when leaving harbor $i$ at arrival $m$ and 0 otherwise,

- $t_{i m c}^{C}$ as the time compartment $c$ starts service in harbor $i$ at arrival $m$, we can rewrite equation (17) to:

$$
\begin{aligned}
\overline{C_{c r}^{C}}= & \sum_{(i, m, j, n) \in A_{v}}\left[\mu_{i m j n}-D_{i m j n c}^{X}\right] x_{i m j n c} \\
& -\sum_{i \in H_{T}} \sum_{m \in M_{T i}}\left[\sum_{g \in G}\left(D_{i m c g}^{V} w_{i m c g}\right)-\sum_{g \in G}\left[D_{i m g}^{Q} q_{i m c g}\right]-D_{i m c}^{S T} t_{i m c}^{C}\right] \\
& -D_{c}^{\lambda}
\end{aligned}
$$

Furthermore, we define $T_{i m p_{g} j n q_{g} c}$ as the minimum time between node $\left(i, m, p_{g}\right)$ and $\left(j, n, q_{g}\right)$ for compartment $c$, based on travel time and (un)loading time. $\left[A_{i m p_{g} c}, B_{i m p_{g} c}\right]$ is the time window for node $\left(i, m, p_{g}\right)$ and $l_{i m p_{g} j n q_{g} c}(t)$ is the latest time for the start of service at node $\left(i, m, p_{g}\right)$, when compartment $c$ directly sails to $\left(j, n, q_{g}\right)$ and starts service within time $t$ :

$$
l_{i m p_{g} j n q_{g} c}(t)=\min \left(B_{i m p_{g} c}, t-T_{i m p_{g} j n q_{g} c}\right)
$$

Next define the recursive function $f_{j n q g c}(t)$ as the minimum costs of compartment $c$ up to arrival at node $\left(j, n, q_{g}\right)$ if the service of this node can start within time $t$ for all $t \in\left[A_{j n q_{g} c}, B_{j n q_{g} c}\right]$ :

$$
\begin{aligned}
f_{j n q_{g} c}(t)=\min _{i, m, p_{g}^{\prime}}\left\{\min _{A_{j n q_{g} c t^{\prime} \leq t}\{}\right. & \mu_{i m j n}-D_{i m j n c}^{X}-D_{i m c g^{\prime}}^{V} \\
& -\left[D_{i m g^{\prime}}^{Q} q_{i m c g^{\prime}}\right]-D_{i m c}^{S T} t^{\prime} \\
& \left.\left.+f_{i m p_{g} c}\left(l_{i m p_{g}^{\prime} j n q_{g} c}\left(t^{\prime}\right)\right)\right\}\right\}
\end{aligned}
$$


with $f_{j n q_{g}}(t)=0$ if $(j, n)$ is the initial position of the ship. Note that $f_{j n q_{g} c}(t)$ is non-increasing in $t$ and that it is piecewise linear. The compartment subproblem is now defined as:

$$
\min _{\left(j, n, q_{g}\right)} f_{j n q_{g} c}(T)
$$

with $T$ the planning horizon.

\subsubsection{Solving the compartment subproblem}

The piecewise linear recursive cost function can be generated by forward propagation. The algorithm, described in section 4.1.3, can be used in a slightly modified form to solve the compartment subproblem.

\section{Concluding Remarks and Further Research}

In this paper, we discussed the multi-product ship routing problem with inventory constraints which arises in the oil industry. Since different oil products cannot be stored in the same compartment of a ship, the capacities of the different compartments of the ship have to be taken into account as well. For this problem, a mixed integer programming problem has been presented in the literature before. However, this could not be used to solve any real-size instances. Therefore, we have developed a branch-and-price algorithm, which has been successfully applied by Christiansen (1999) for a the single-product ship routing problem with inventory constraints. We have extended her set partitioning type of formulation to the case of multiproducts, where different grades of oil have to be transported in different compartments.

One of the purposes of this paper is to present the ship routing problem in the oil industry to the OR community. We would like to encourage other researchers to work on this problem. In addition, we hope that practitioners from the oil industry to use our method to solve practical problem instances. Many practical aspects such as grades that have to be transported in special compartments can be directly incorporated into our mathematical model and solution approach.

However, further research has to be done on including stochastic travel times and cyclic schedules. Due to weather conditions and the streams in the ocean, a ship can often have longer or shorter travel times than scheduled. Furthermore, cyclic schedules are usually preferred by the refineries of the oil company such that they can easily schedule their staff. 


\section{References}

Al-Khayyal, F., \& Hwang, S.J. 2007. Inventory constrained maritime routing and scheduling for multi-commodity liquid bulk, Part I: Applications and model. European Journal of Operations Research, 176, 106-130.

Brønmo, G., Nygreen, B., \& Lysgaard, J. 2009. Column generation approaches to ship scheduling with flexible cargo sizes. European Journal of Operations Research, 200, 139-150.

Christiansen, M. 1999. Decomposition of a combined inventory and time constraint ship routing problem. Transportation Science, 33, 3-16.

Christiansen, M., \& Nygreen, B. 1998. A method for solving ship routing problems with inventory constraints. Annals of operations research, 81, $357-378$.

Christiansen, M., \& Nygreen, B. 2005. Robust inventory ship routing by column generation. Pages 197-224 of: Desaulniers, G., Desrosiers, J., \& Solomon, M.M. (eds), Column Generation. Springer, New York.

Christiansen, M., Fagerholt, K., Nygreen, B., \& Ronen, D. 2004. Maritime Transportation. Pages 35-139 of: Barnhart, C., \& Laporte, G. (eds), Transportation. Handbooks in Operations Research and Management Science, vol. 8. North-Holland, Amsterdam.

Desaulniers, G., Desrosiers, J., \& Solomon, M.M. (eds). 2005. Column Generation. Springer, New York.

Desrosiers, J., Dumas, Y., Solomon, M.M., \& Soumis, F. 1995. Time Constrained Routing and Scheduling. Pages 35-139 of: Ball, M.O., Magnanti, T.L., Monma, C.L., \& Nemhauser, G.L. (eds), Network Routing. Handbooks in Operations Research and Management Science, vol. 8. NorthHolland.

Ronen, D. 2002. Marine inventory routing: shipments planning. Journal of the Operations Research Society, 53, 108-114. 